\title{
Direct visualization of conformation and dense packing of DNA-based soft colloids
}

\author{
Jing Zhang ${ }^{1}$, Paul M. Lettinga ${ }^{1,2}$, Jan K. G. Dhont ${ }^{1,3}$, and Emmanuel Stiakakis ${ }^{1, *}$ \\ ${ }^{1}$ Forschungszentrum Jülich, Institute of Complex Systems 3, Leo-Brandt-Str, 52425 Jülich, Germany \\ ${ }^{2}$ Department of Physics and Astronomy, Laboratory for Acoustics and Thermal Physics, Katholieke \\ Universiteit Leuven, Celestijnenlaan 200D, Leuven B-3001, Belgium \\ ${ }^{3}$ Heinrich-Heine-Universität Düsseldorf, Universitätsstraße 1, 40225 Düsseldorf, Germany \\ * Corresponding Author: e.stiakakis@fz-juelich.de
}

\begin{abstract}
Soft colloids - such as polymer-coated particles, star polymers, block-copolymer micelles, microgels - constitute a broad class of materials where microscopic properties such as deformability and penetrability of the particle play a key role in tailoring their macroscopic properties which is of interest in many technological areas. The ability to access these microscopic properties is not yet demonstrated despite its great importance. Here we introduce novel DNA-coated colloids with star-shaped architecture that allows accessing the above local structural information by directly visualizing their intramolecular monomer density profile and arm's free-end locations with Confocal Fluorescent Microscopy. Compression experiments on a two-dimensional hexagonal lattice formed by these macromolecular assemblies reveal an exceptional resistance to mutual interpenetration of their charged corona at pressures approaching the $\mathrm{MPa}$ range. Furthermore, we find that this lattice, in a close packing configuration, is surprisingly tolerant to particle size variation. We anticipate that these stimuliresponsive materials could aid to get deeper insight in a wide range of problems in soft matter including the study and design of biomimetic lubricated surfaces.
\end{abstract}


Charged polymeric layers densely attached by one end to an interface, also known as polyelectrolyte brushes, are systems that exhibit rich and complex behavior [1-7] with direct relevance to biological systems [8-11] and with a diverse range of applications such as surface modification technologies $[10,12,13]$ and emerging biotechnologies $[12,14,15]$. Experimental observations of charged brushes available at present are not so sensitive to the details of the structure but are mainly concerned with more global brush properties, such as brush thickness [16-18] or force-distance dependence between brushes [19-21]. However, the detailed picture of a polyelectrolyte brush comprises the determination of intramolecular density profiles and freeend arm distributions for the case of a dilute, non-interacting system. In the case of crowded conditions additional parameters have to be quantified, namely how the grafted charged coronas of brushes interact in terms of shrinkage, deformation or penetration. In particular for the crowded case, many of the above mentioned parameters are poorly accessible in a variety of physically distinct soft and penetrable colloidal systems [22-25]. In this Letter, we show how the above issues can be addressed by grafting very long double-stranded DNA (dsDNA) chains to the surface of a micron-sized spherical super-paramagnetic particle for the construction of strongly charged, ultra-dense, highly monodisperse PolyElectrolyte brushes with a star-shaped architecture (abbreviated by PE-star hereafter, see Supplemental Material (SM) [26] for details on the synthetic strategy, section 1.1-1.2).

Direct visualization of these water soluble DNA-based microstructures is achieved by fluorescent staining either the whole DNA chain (Backbone Labeling, BL) or only its free-end (Free-End Labeling, FEL). In particular, the second labeling strategy allowed us to directly observe the internal structure of single of PE-star under highly crowded conditions, which is a significant improvement to existing visualization methods [33,34]. The above two labelling 
approaches are depicted schematically in Figure 1a-b together with their corresponding Confocal Fluorescence Microscopy (CFM) images taken at their equatorial plane under dilute salt-free conditions (see SM [26], section 1.3 for details on the labeling method and the Supporting Movie shows the 3D reconstruction of a fluorescence free-end labeled PE-star based on confocal microscopy sections). The corona thickness $L$ of a PE-star can be extracted from the azimuthally averaged radial fluorescent intensity of the above CFM images. Such a profile is shown in the top inset of Figure 1c for the BL case. A power-law fit (Figure 1c) of the thickness $L$, as extracted from the corresponding CFM images presented in Figure 1d, versus the arm length $N$ for fixed $f$ - number of arms (termed functionality) yields $L \sim N$ under salt-free conditions. In addition, we find that $L$ equals to the contour length $L_{c}$ of the grafted dsDNA fragments, which indicates an extreme arm stretching while $L$ is practically $f$-independent over a wide range of arm lengths $N$ (bottom inset Figure 1c). This conformational behavior of the stars - owing to their ability to act as an efficient absorber of its own counterions under salt-free condition $[2,35-$ $38]$ - is in excellent agreement with the scaling predictions for an osmotic star [2,35].

Information on the internal star structure is extracted from the azimuthally averaged mass density profile, which is directly related to the radial fluorescent intensity of the BL PE-star [39]. A density profile is shown in Figure $2 \mathrm{a}$ for an isolated osmotic (salt-free) PE-star with $f=48 \mathrm{~K}$ and $N=30 \mathrm{Kbp}$. Since the corona thickness follows the scaling $L \sim N$, the extension of the arms of PE-star is expected to be uniform. In a spherical geometry, this means that the radial distribution of the mass density in the star decays as $r^{-2}$, where $r$ is the distance from the center of the star. Indeed, the slope of the measured profile in log-log representation is found to be very close to -2 , supporting the picture of fully stretched chains having rod-like configuration [40-42,33]. By adding salt, the measured density profile reveals rearrangement of the intrinsic structure of the 
osmotic PE-star. More specific, the significant de-swelling of the PE-star, as demonstrated in the CFM images (Figure 2a), is accompanied by a change of the slope of the mass density profile from around -2 in the salt-free regime to $-4 / 3$ at relatively high salt concentration. The latter power law decay implies conformational behavior similar to a neutral star with enlarged (electrostatic) excluded volume, which has been predicted for the salted regime $[41,43]$.

Complementary insight into the osmotic (salt-free) to salted regime transition can be obtained from the free-end distribution, as reflected from the azimuthally averaged radial fluorescent intensity of CFM images of FEL PE-stars (Figure 2b, see also SM [26], section 1.4 regarding the gradual nature of this transition as reflected from the changes in the size of the PE-star). While the free-end distribution confirms the thickness of osmotic PE-star corona as found from the BL counterpart, it displays a maximum with narrow width, located at the periphery of the star (Figure $2 \mathrm{~b}$, red-circles) with its position being virtually independent of the functionality. As a consequence, a region close to the center of the star is observed, where the free-ends are depleted ("dead-zone", blue arrow, Figure 2b). By increasing the salt concentration, the free-end distribution becomes wider and its maximum moves to the center of the PE-star (Figure 2b, black-circles) indicating star shrinkage (see SM [26], section 1.4 for more details). Interestingly, the width of the "dead-zone" relative to the size of the PE-star decreases substantially with the addition of the salt and finally disappears for lower functionality PE-star (Figure 2b, triangles). This is an additional verification for the similarity between neutral and highly salted stars, where excluded volume interactions play a dominant role in the equilibrium conformational structure of these macromolecular assemblies.

So far we focused on the conformational properties of an isolated PE-star. We now consider PE-star accommodation scenarios under highly crowded conditions for star concentrations above 
the close packing fraction of spherical, undeformable objects with the same size as the isolated star. A simple and robust approach was employed for achieving tunable crowded conditions, utilizing a millimeter-sized square permanent magnet and taking advantage of the superparamagnetic properties of star's core (Figure 3a, see also SM [26], section 2.1 for more details). Control over the magnet position $h$, thickness $s$ of the sample holder and particle concentration provides an easy and robust way to organize the PE-stars into two-dimensional (2D) hexagonal ordered arrays and subsequently compress them from an open (Figure 3b) to space-filling configuration (Figure 3c).

Visual inspection of Figure $3 \mathrm{c}$ suggests that the space-filling lattice configuration is accompanied with the loss of the PE-star spherical symmetry and not by arm interpenetration. Unambiguous evidence is provided by the CFM image in Figure $3 \mathrm{~d}$ showing a compressed 2D crystal formed by osmotic PE-stars $(f=336 \mathrm{~K}$ and $N=20 \mathrm{Kbp})$ where a few FEL tracer PE-stars are embedded in a host environment of unlabeled stars. A 2D hexagonal arrangement is achieved which is just space-filling. This is verified by the sharp facetted labeled PE-star, the relative position of star magnetic cores and the fact that the distance $L^{*}$ from the surface of the core to the vertices equals the corona radius of the undisturbed star $L$ (inset of Figure $3 \mathrm{~d}$ ). The above observations indicate that the free-ends are located near the facets with no observable interpenetration. The same behavior is even observed for stars with much lower functionality $(f=41 \mathrm{~K}$ and $N=20 \mathrm{Kbp}$, see $\mathbf{S M}[26]$, section 2.2.1).

The CFM images (Figure 3d and Figure S2 [26]) provide direct evidence for noninterdigitation of the arms from different osmotic PE-stars. Non-interdigitation is the main assumption of Pincus's theory [2], based on scaling arguments, on the interactions between PEstars which arises mainly from the entropic contribution of the trapped counterions [37]. In the 
salt-dominated regime for stars with low functionality $(f=69 \mathrm{~K})$ we do observe intra-arm penetration to some extent (Figure 3e, also see SM [26], section 2.2.2). However, in both cases the arms of two PE-stars cannot penetrate more than the width of their free-end distribution. The latter observation has strong implications for using polyelectrolytes as a biomimetic lubrication agent between surfaces, since the degree of arm penetration controls entanglements which in turn influence the friction between compressed PE-stars.

We estimate the pressures involved in the above compression experiments on the $2 \mathrm{D}$ hexagonal PE-star lattice by conducting osmotic stress experiments on an isolated salt-free PEstar using neutral polymer dextran as an osmotic stress agent (see SM [26], section 2.3). In particular, assuming that the radius of the incircle of the hexagonal compressed PE-star is equivalent to the radius of an osmotic compressed PE-star then the compression in Figure $3 \mathrm{~d}$ corresponds to a pressure of $8.5 \mathrm{KPa}$ (red-cross in Figure S5 [26]). A higher degree of magnetically-mediated PE-star compression has been achieved by employing a similar approach as the one depicted in Figure 3a, but now close to a water-air interface (see SM [26], section 2.2.3). The results show a load carrying ability of the osmotically counterion swollen PE-stars without any detectable interpenetration of their arms at pressures well above the highest measurable osmotic pressure of $25 \mathrm{KPa}$. Therefore, our observations reveal that the osmotic pressure due to the trapped counterions inside the PE-star corona is indeed the primary mechanism for exceptional lubrication properties of polyelectrolyte brushes up to very high normal pressures as suggested in Ref. 5, 10, 44.

It is reasonable to hypothesize that a $2 \mathrm{D}$ PE-star crystal may be more tolerant to the inclusion of particles of different sizes given the PE-star deformability, stimuli-responsiveness and remarkable resistance to interpenetration of their charged coronas as they are packed more 
densely. This is in contrast to a 2D hard-sphere colloidal crystal [45] or any deformable but volume preserving system such as $2 \mathrm{D}$ bubble rafts [46]. The effects of the inclusion of a larger PE-star are illustrated with the CFM image of Figure 4a. A 2D close-packed crystal under mild salinity conditions formed by FEL PE-stars was doped with unlabeled larger PE-stars with a volume 3.4 times that of the host particles. Interestingly, the oversized doped star fits into the host structure without causing local distortion of the crystal lattice. This is evidenced by a comparison of the CFM image of Figure 4b, where only the doped PE-stars are labeled, with the corresponding bright field confocal microscopy image in Figure 4c, where only the magnetic cores of the host and foreign particles are visible. Indeed, the doped particles adapt to their new local environment by becoming highly compressed and facetted as they are squeezed by neighboring PE-stars. The final shape of the oversized foreign particles in the $2 \mathrm{D}$ crystal is dictated by the number and type of first nearest neighbors. Thus, in our case, the shape of the doped particle is found to be a hexagon with concave edges (Figure $4 \mathrm{~b}$ and inset) or a mixture of concave and flat edges (as indicated by arrows in Figure 4b) depending on whether it is surrounded by only host or a mixture of host and doped particles, respectively.

Comparison of the foreign particle dimensions with and without confinement (blue circle in Figure $4 \mathrm{~b}$ ) suggests that the doped particles adjust their volume not only because of direct interactions between the particles but also through a shrinking pathway. The latter mechanism was also reported in similar experiments performed on three-dimensional crystals made of micrometer-sized soft microgels particles, where this behavior is attributed solely to osmotic effects [47]. In our case, the ability to visualize local structural features with high resolution reveals that a delicate synergy exists between the osmotic pressure within each particle and the elastic deformation of its charged corona. Additional support has been obtained by exploring the 
role of salt and size ratio in the impurity-tolerance of a 2D PE-star crystal. The results show the existence of a narrow window of parameters where the integration of larger particle is possible (see SM [26], section 2.4), stressing the role of softness of the interaction potential on the defect tolerance of our 2D hexagonal PE-crystals. A rich phase behavior of PE-star solutions based on effective interactions [48] has been predicted and partially experimentally verified with SAXS measurements [49]. Our work suggests that a refinement of the models used for the predictions is at place, taking into account density-dependent particle shrinkage and deformation. This may reveal even more unusual phase behavior.

In summary, we have demonstrated an easy and robust route for the fabrication of ultra-dense long dsDNA-coated super-paramagnetic particles. The resulting star-branched polyelectrolytes allow for the direct visualization of the internal structure, deformability and interpenetrability of these model charged soft-spheres under dilute and highly packed conditions at an unprecedented level of detail. Our results on their exceptional resistance to mutual interpenetration, at pressures approaching the MPa range, could be a great value for "in-situ" study of tribological processes involving polymeric surfaces in aqueous environments. Moreover, our approach facilitates the simultaneous measurement of friction forces and direct visualization of the brush, which could have a significant impact on the design of new bio-lubrication strategies $[10,15]$.

\section{Acknowledgements}

The research was supported by the Transregio Sonderforschungsbereich TR6018 'Physics of Colloidal Dispersions in External Fields'. 


\section{References}

[1] S. Misra, S. Varanasi, and P. P. Varanasi, Macromolecules 22, 4173 (1989).

[2] P. Pincus, Macromolecules 24, 2912 (1991).

[3] O. V. Borisov, E. B. Zhulina, and T. M. Birshtein, Macromolecules 27, 4795 (1994).

[4] M. Ballauff and O. V. Borisov, Curr. Opin. Colloid Interface Sci. 27, 316 (2006).

[5] J. B. Sokoloff, J. Chem. Phys. 129, 014901 (2008).

[6] A. Jusufi and C. N. Likos, Rev. Mod. Phys. 81, 1753 (2009).

[7] O. V. Borisov, E. B. Zhulina, F. A. M. Leemakers, M. Ballauff, and A. H. E. Müller, Adv. Polym. Sci. 241, 1 (2011).

[8] E. B. Zhulina and F. A. M. Leemakers, Soft Matter 5, 2836 (2012).

[9] A. Dédinaite, Soft Matter 8, 273 (2012).

[10] A. Raviv, S. Giasson, N. Kampf, J. F. Gohy, R. Jérôme, and J. Klein, Nature 425, 163 (2003).

[11] S. S. Daube, D. Bracha, A. Buxboim, and R. H. Bar-Ziv, Proc. Natl. Acad. Sci. U.S.A. 107, $2836(2010)$.

[12] A. Wittemann, B. Haupt, and M. Ballauff, Phys. Chem. Chem. Phys. 5, 1671 (2003).

[13] F. Zhou, P. M. Biesheuvel, E. Y. Choi, W. Shu, R. Poetes, U. Steiner, and W. T. Huck, Nano Lett. 8, 725 (2008).

[14] D. J. Lockhart, H. Dong, M. C. Byrne, M. T. Follettie, M. V. Gallo, M. S. Chee, M. Mittmann, C. Wang, M. Kobayashi, H. Horton, and E. L. Brown, Nature Biotechnology 14, 1675 (1996).

[15] T. Moro, Y. Takatori, K. Ishihara, T. Konno, Y. Takigawa, T. Matsushita, U. Chung, K. Nakamura, and H. Kawaguchi, Nature Mater. 14, 829 (2004). 
[16] R. Hariharan, C. Biver, J. Mays, and W. B. Russel, Macromolecules 31, 7506 (1998).

[17] X. Guo and M. Ballauff, Phys. Rev. E 64, 051406 (2001).

[18] A. S. Lee, V. Bütün, M. Vamvakaki, S. P. Armes, J. A. Pople, and A. P. Gast, Macromolecules 35, 8540 (2002).

[19] K. Kegler, M. Konieczny, G. Dominguez-Espinoza, C. Gutsche, M. Salomo, F. Kremer, and C. N. Likos, Phys. Rev. Lett. 100, 118302 (2008).

[20] G. Dominguez-Espinoza, A. Synytska, A. Drechsier, C. Gutsche, K. Hegler, P. Uhlmann, M. Stamm, and F. Kremer, Polymer, 49, 4802 (2008).

[21] F. Huang, K. Addas, A. Ward, N. T. Flynn, E. Velasco, M. F. Hagan, Z. Dogic, and S. Fraden, Phys. Rev. Lett. 102, 108302 (2009).

[22] C. M. Hui, J. Pietrasik, M. Schmitt, C. Mahoney, J. Choi, M. R. Bockstaller, and K. Matyjaszewski, Chem. Mater. 26, 745 (2014).

[23] J. Roovers, Macromolecules 27, 5359 (1994).

[24] J. Buitenhuis and S. Förster, J. Chem. Phys. 107, 262 (1997).

[25] H. Senff and W. Richtering, J. Chem. Phys. 111, 1705 (1999).

[26] See Supplemental Material [url], which includes Refs. [27-32].

[27] K. Kegler, M. Salomo, and F. Kremer, Phys. Rev. Lett. 98, 058304 (2007).

[28] A. Buxboim, S. S. Daube, and R. Bar-Ziv, Nano Lett. 9, 909 (2009).

[29] C. Carlsson, M. Johnson, and B. Akerman, Nucleic Acids Res. 23, 2413 (1995).

[30] A. L. Gassner, M. Abonnenc, H. X. Chen, J. Morandini, J. Josserand, J. S. Rossier, J. M. Busnel, and H. H. Girault, Lab Chip 9, 2356 (2009).

[31] F. R. Senti, N. N. Hellman, N. H. Ludwig, G. E. Babcock, R. Tobin, C. A. Glass, and B. L. Lamberts, J. Polym. Sci. 17, 527 (1995). 
[32] V. A. Parsegian, R. P. Rand, N. L. Fuller, and D. C. Rau, Methods Enzymol. 127, 400 (1986).

[33] A. Wittemann, M. Drechsler,Y. Talmon, and M. Ballauff, J. Am. Chem. Soc. 127, 9688 (2005).

[34] F. A. Plamper, A. Walther, A. H. E. Müller, and M. Ballauff, Nano Lett. 7, 167 (2007).

[35] O. V. Borisov, J. Phys. II (France) 14, 1 (1996).

[36] M. Roger, P. Guenoun, F. Muller, L. Belloni, and M. Delsanti, Eur. Phys. J. E. 9, 313 (2002).

[37] A. Jusufi, C. N, Likos, and H. Löwen, J. Chem. Phys. 116, 11011 (2002).

[38] F. A. Plamper, H. Becker, M. Lanzendörfer, M. Patel, A. Wittemann, M. Ballauff, and A. H. E. Müller, Macromol. Chem. Phys. 206, 1813 (2005).

[39] Our CCD camera is non-intensified, allowing for a linear relationship between the intensity of light impinging on a pixel and the final value of the digital intensity in the acquired image. Since the BL labeling method involves intercalation of fluorescent dye which is insensitive to base pair sequence, it is valid to assume that the fluorescent intensity is linearly related with DNA mass at a particular image location. Therefore, the fluorescent intensity decay can be directly related to the radial dependence of the star mass density profile.

[40] O. V. Borisov and E. B. Zhulina, J. Phys. II 7, 449 (1997).

[41] O. V. Borisov and E. B. Zhulina, Eur. Phys. J. B. 4, 205 (1998).

[42] P. Guenoun, F. Muller, M. Delsanti, L. Auvray, Y. J. Chen, J. W. Mays, and M. Tirell, Phys. Rev. Lett. 81, 3872 (1998). 
[43] J. Klein Wolterink, F. A. M. Leermakers, G. J. Fleer, L. K. Koopal, E. B. Zhulina, and O. V. Borisov, Macromolecules 32, 2365 (1999).

[44] U. Raviv, S. Giasson, N. Kampf , J. F. Gohy, R. Jérôme, and J. Klein, Langmuir 24, 8678 (2008).

[45] D. R. Nelson, M. Rubinstein, and F. Spaepen, Philos. Mag. A 46, 105 (1982).

[46] L. Bragg and J. F. Nye, Proc. R. Soc. Lond. A 190, 474 (1947).

[47] A. S. Iyer and A. L. Lyon, Angew. Chem. Int. Edit. 48, 4562 (2009).

[48] N. Hoffmann, C. N. Likos, and H. Löwen, J. Chem. Phys. 121, 7009 (2004).

[49] T. Furukawa and K. Ishizu, Macromolecules 38, 2911 (2005). 


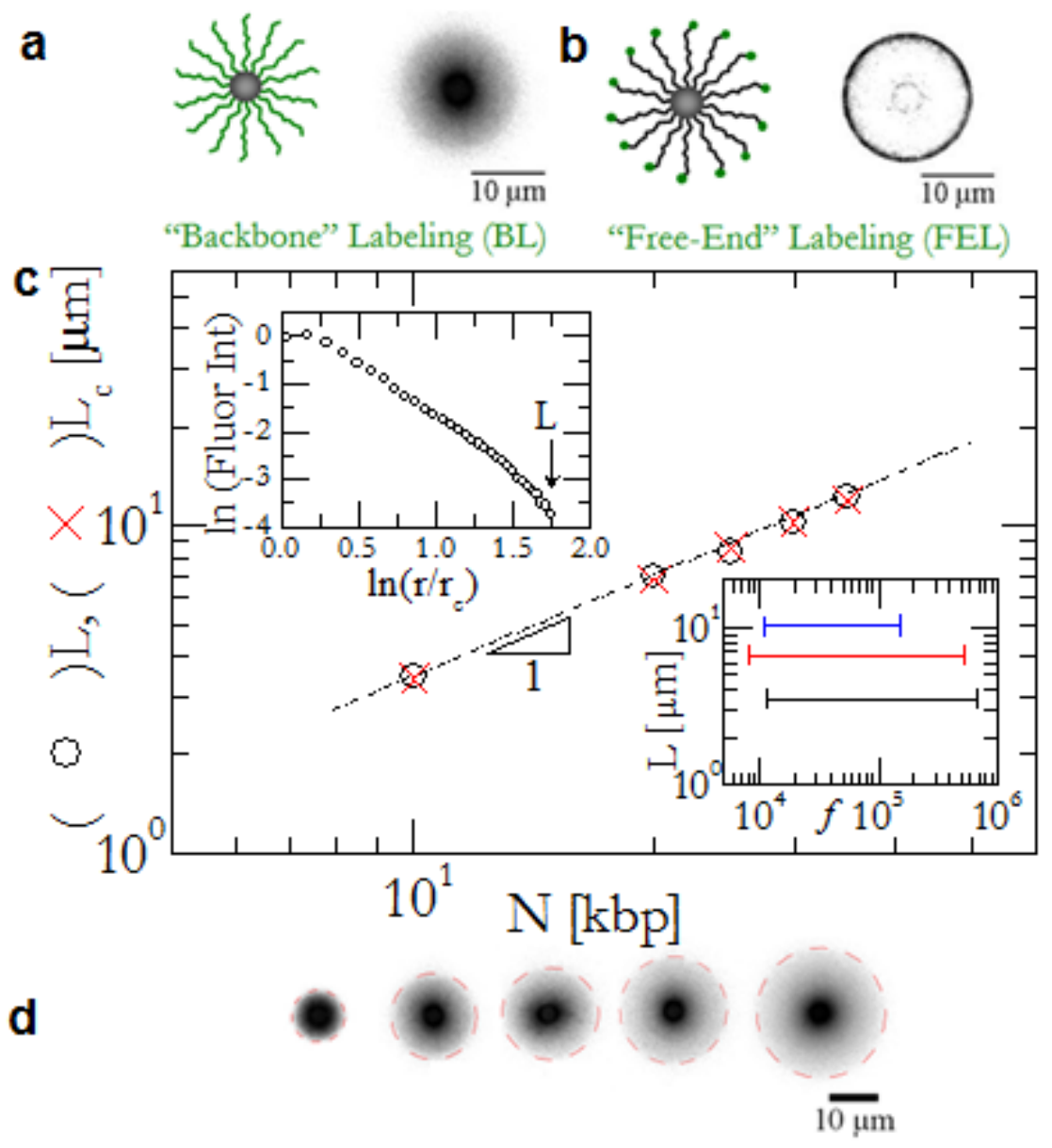

Figure 1. a-b, Cartoon of the two fluorescent labeling strategies (shown in green) employed for the direct visualization of the DNA-based PE-stars with their corresponding CFM images at their equatorial plane of particles (radius $r_{c}=1.44 \mu \mathrm{m}$ ) grafted with $300 \mathrm{~K}$ dsDNA chains of $20 \mathrm{~K}$ base pairs (bp). c, Osmotic star corona thickness $L$ (circles) versus the arm length $N$ with $f=140 \mathrm{~K}$. Red crosses indicate the contour lengths $L_{c}$ of the star arms. The line is the prediction of the scaling law for osmotic PE stars. Top inset: azimuthally averaged radial fluorescent intensity (normalized to the intensity on the surface of the star's core) versus the distance $r$ (normalized to radius $r_{c}$ of the core) from the center of a salt-free PE-star with $f=89 \mathrm{~K}$ and $N=20 \mathrm{Kbp}$. Bottom inset: the explored regions of functionalities where the star's corona thickness is independent of $f$ (black, red, blue line: $N=10,20,30 \mathrm{Kbp}$ ). d, CFM images at the equatorial plane of the osmotic 
PE-stars with $f=140 \mathrm{~K}$ and variable arm size (Left to right: $N=10,20,25,30,35 \mathrm{Kbp}$ ). The red circles indicate the size of PE-star. 


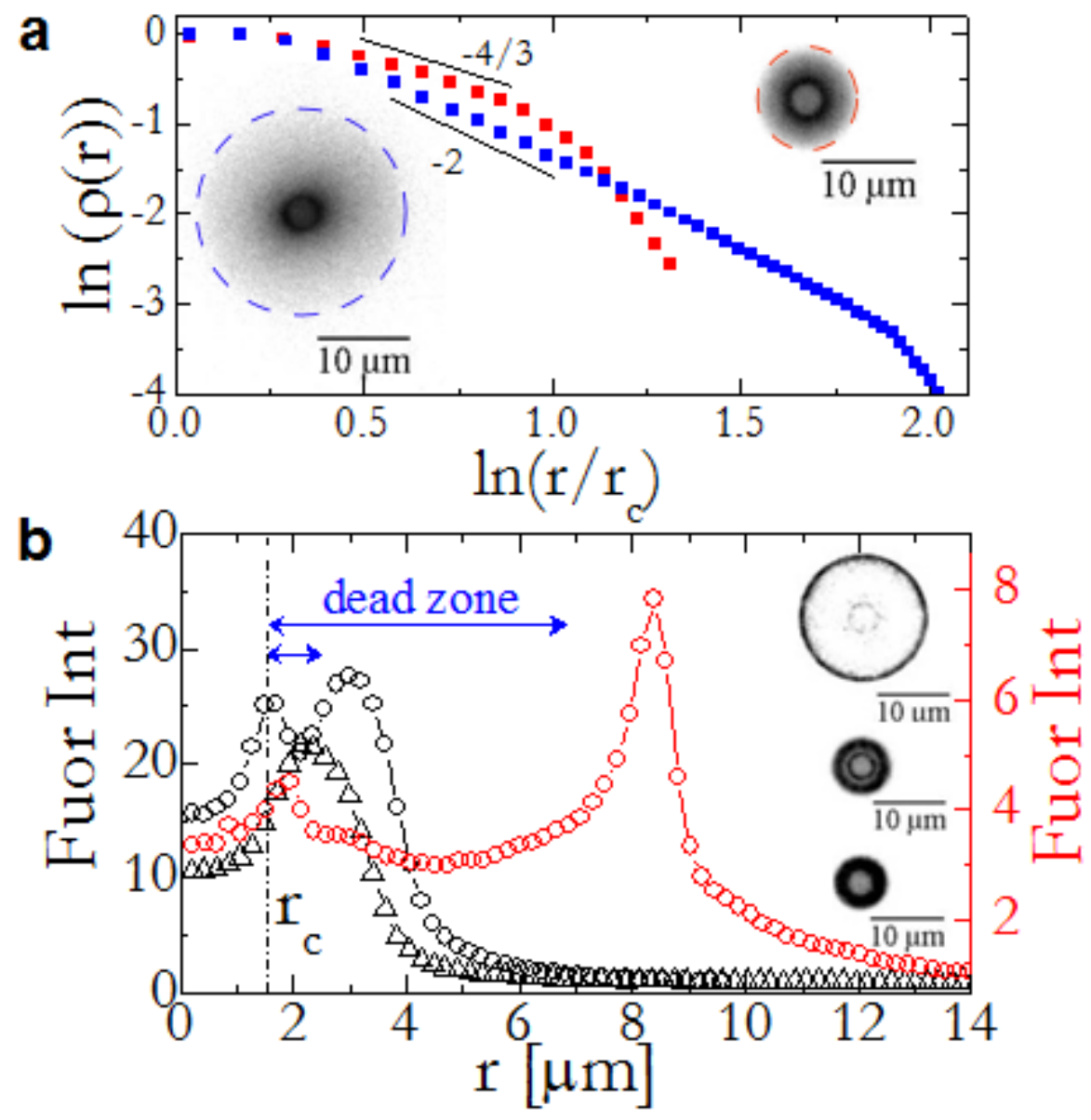

Figure 2. a, DNA mass density profile $\rho(r)$ versus the distance $r$ (normalized to radius $r_{c}$ of star's core) from the center of a BL PE-star with $f=48 \mathrm{~K}$ and $N=30 \mathrm{Kbp}$ under different salinity conditions together with their corresponding CFM images (salt-free: blue squares, bottom image and $0.1 \mathrm{M} \mathrm{NaCl}$ : red squares, top image. The circles indicate the size of PE-star). The bold lines show the slopes predicted by scaling. b, The azimuthally averaged fluorescent intensity of CFM images of FEL PE-star (normalized to the background fluorescent intensity) versus $r$ for salt-free $(f=514 \mathrm{~K}, N=20 \mathrm{Kbp}$, red circles, top image), $1.0 \mathrm{M} \mathrm{NaCl}(f=514 \mathrm{~K}, N=20 \mathrm{Kbp}$, black circles, middle image), and $1.0 \mathrm{M} \mathrm{NaCl}(f=131 \mathrm{~K}, N=20 \mathrm{Kbp}$, triangles, bottom image). The position of the surface of the star's core is indicated with a black line. 

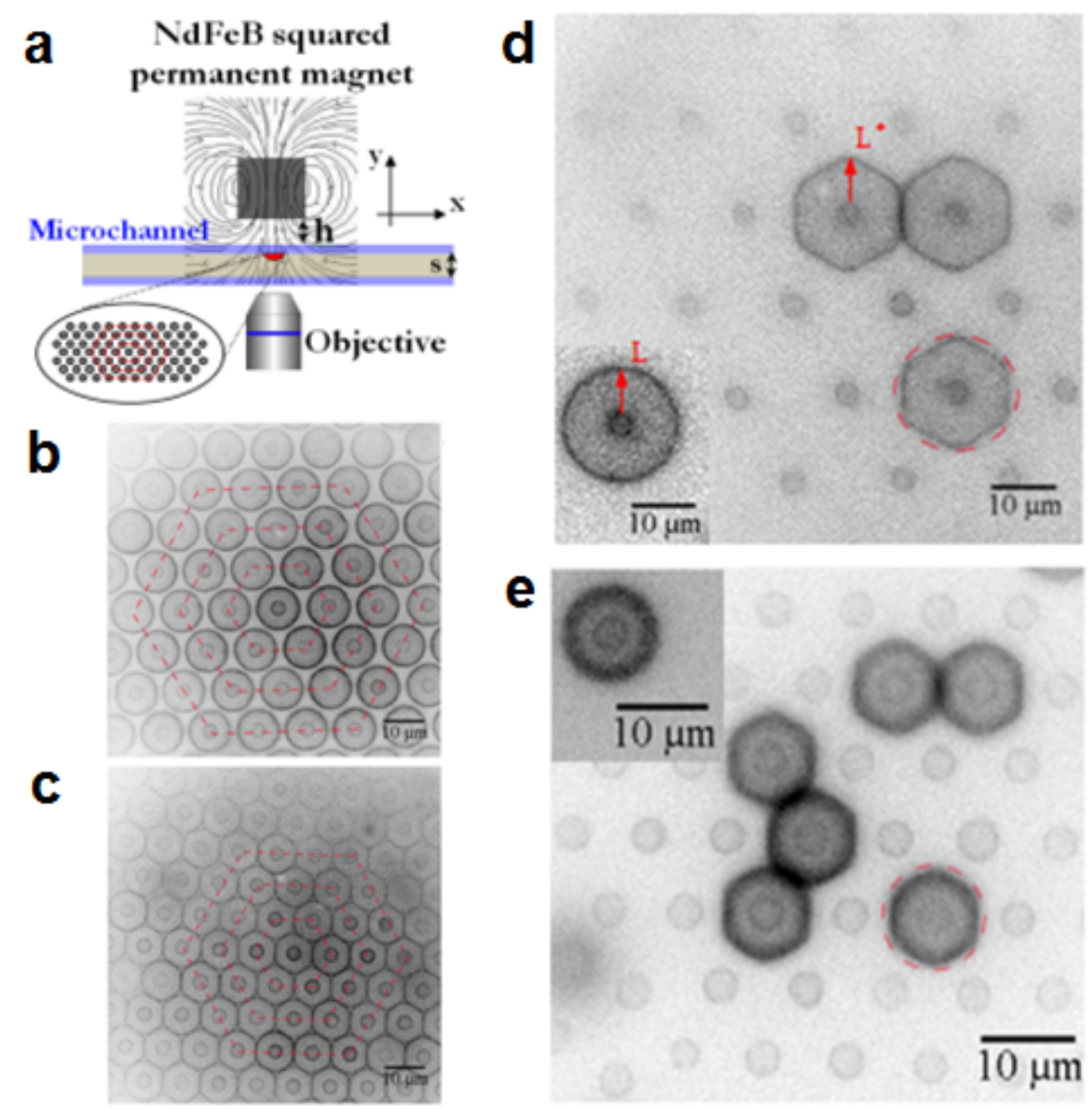

Figure 3. a, Schematic diagram of the magnetic PE-star hexagonal array guidance system. b-d, CFM images of 2D hexagonal lattice formed by the magnetically-mediated self-assembly of FEL labeled PE-stars with b, open and c-e, space filling configuration. b-c, Osmotic stars with $f=467 \mathrm{~K}$ and $N=10 \mathrm{Kbp}$. The red hexagons indicate the formation and homogeneous compression of the 2D PE-star lattice. d, Osmotic stars with $f=336 \mathrm{~K}$ and $N=20 \mathrm{Kbp}$. e, Mild-salted (5mM $\mathrm{NaCl})$ stars with $f=69 \mathrm{~K}$ and $N=20 \mathrm{Kbp}$. For d-e only a small fraction of PE-stars are FEL labeled. The corresponding CFM images for isolated PE-star under the same salinity conditions are displayed in the insets. The red circles indicate the size of a PE-star in the undisturbed state (see insets), where $L$ is the corona thickness. $L^{*}$ is the distance from the surface of the core of the hexagonal-shaped PE-star to its vertex. 


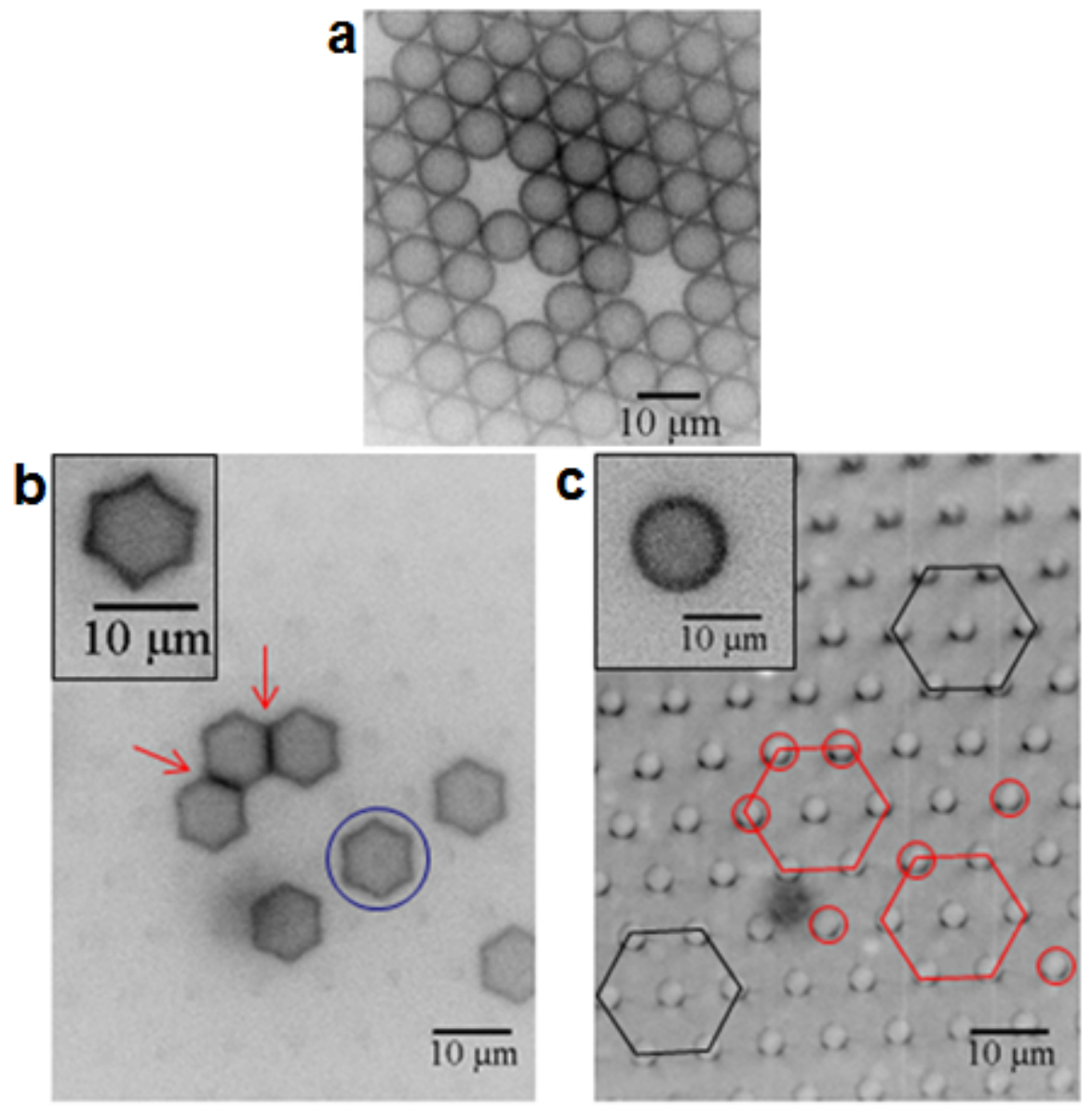

Figure 4. Confocal microscopy images of a closed packed 2D hexagonal lattice formed by PEstars $(f=310 \mathrm{~K}$ and $N=10 \mathrm{Kbp})$ that can tolerate the presence of larger foreign PE-stars $(f=391 \mathrm{~K}$ and $N=20 \mathrm{Kbp})$ under mild salinity conditions $(5 \mathrm{mM} \mathrm{NaCl})$. a-b, CFM images where only the host or foreign PE-stars are labeled (FEL), respectively. The blue circle indicates the size of an undisturbed doped star. Inset: an oversized doped PE-star with highly curved edges. The red arrows point to the flat edges. c, Bright field image corresponding to CFM image of (b), where only the magnetic cores of the PE-stars (host and foreign) are visible. The red circles indicate the location of the foreign particles. Visual comparison of the black and red hexagons, which contain only host and mixture of host and foreign PE-stars respectively, suggests that the crystal lattice is not affected by the presence of large particles. Inset: Undisturbed foreign PE-star. 